\title{
Mirror Neurons Underpinnings of Communication Aspects: Inter-subjectivity and Socio-cultural Dimensions
}

\author{
Baaziz Termina \\ Mohamed V University, Rabat, Morocco
}

\begin{abstract}
The central aim of this article is to account for communication's diverse behavioral aspects: actional, motional, audio-visual, emotional, and intentional and their neural underpinnings. Based on neural substrates, the aforementioned aspects will be illuminated in terms of their interpersonal and socio-cultural dimensions active in human interaction. Culture in this context is to be approached, on the one hand, as a guideline identifying a given group or society in a given neuro-social space and taken, on the other hand, as a universal catalogue of synchronized human behavior. The framework we draw on our lines of reasoning incorporates Mirror Neurons theory and Neural Networks conception, equally referring to an interdisciplinary-grounded perspective.
\end{abstract}

Keywords: Mirror Neurons, Neural Networks, communication, understanding, behavior, interconnectedness

\section{Introduction}

Since the ground breaking exploration of what has been proven to be Mirror Neurons System, there has been an exploding interest in the topic as the literature reveals. The bulk of seminal work revolves around the core tenet that the same neurons fire when performing an action, observing another person performing it, or even hearing its commensurable sound. This basic fact, discovered first in Macaque, has blossomed into plethora of promising speculations on the involvement of mirror neurons circuits in a wide range of inter-related mental states and behaviors, including those related to the neural interconnectedness among individuals.

Mirror Neurons theory is hypothesized to have been influenced by Hebbian rule. In his book The Organization of Behavior (1949), Donald Hebb lays out the connectionist ground for discerning how neural networks learn to adapt during learning process. Constituting the mainstay of connectionist theories and synaptic plasticity (Brown \& Milner, 2003), he postulates that every experience one encounters modulates his neural networks. He attributes learning to synaptic plasticity that enables neurons to connect and make auto-associations, forming cell assemblies. Hebb (1949) dexterously elucidates how persistent activation of interconnected neurons strengthens their synaptic efficacy through increasing their weight. This rule is summed up by Siergrid Lowels' (1992) reiterated phrase: "cells that fire together, wire together".

Hebbian rule, which is known in the currently drawn literature as "Spike Timing Dependent Plasticity", is hypothesized to have influenced the theoretical investigation of how Mirror Neurons System Emerges (Keysers, 2004, 2011) on the basis that mirror neurons are those cells which, by means of auto-associative learning, learn

Baaziz Termina, Professor of English and Ph.D. student, Comparative Linguistics, Institute for Studies and Research on Arabization, Mohamed V University.E-mail: terminabaziz@gmail.com. 
to fire together when we perform, see, hear, or feel a particular action (Gallese, Fadiga, Fogassi, \& Rizzolatti, 1996). In communication, building on firing and hiring rule, mirror neurons are active to feel what the others feel and understand their intentions behind their verbal acts and gestural clues as a broad body of seminal literature on the topic reveals.

The lines of argumentation of the article are as follows:

(1) Mirror neurons involvement in interpersonal communication;

(2) Mirror neurons as a common code: inter-subjectivity in intra-cultural space;

(3) Communication and mirror neurons: universal dimension.

\section{Mirror Neurons Involvement in Communication: Interpersonal Dimension}

Derived from the Latin word communis, which means common, communication is defined by Keyton (2011) as the process of transmitting information and common understanding from one person to another (see Anthony, 2016); another definition refers to verbal and nonverbal exchange of thoughts and emotions (Schiaratura, Pastena, Askevis-Leherpeux, \& Clément, 2015). Language and nonverbal acts operate together in a synchronized way in communication. Language rhythm is synchronized with the rhythm of body movement. The nonverbal components may be in accordance with the linguistic message or contradictory to it (Key, 1973). Investigating the underpinning of gestural communication, Rizzolatti (2004) claims that "nonverbal" or gestural communication' mirror neurons represent neurophysiological substrates of a mechanism that establishes a direct line between the sender of a given message and its recipient. He observes that this mechanism turns the performed actions into "messages that are understood by an observer without any cognitive mediation" since it "creates a common, non-arbitrary, semantic link" between the communicating individuals.

Rizzolatti's investigation, as he construes, resonates with the evidences provided by other authors who emphasize the existence of a link between object-directed and communicative action (See Doran \& McNeilage, 1998). Along this line of reasoning, Rizzolatti reveals that "semantics is inherent to the gestures used to communicate" a given message, which might be explained by the fact that hand/arm and speech are interconnected (Rizzolatti, 2004). They share a common neural substrate, that is, co-activation of the dominant hand motor cortex and higher levels of language (Meister et al., 2003; Rizzolatti, 2004). In addition to communication of gestures and actions, data show further links between the neural underpinning of actions understanding and change inhered in actions and vision.

According to Schubotz and Von Cramon's (2001, 2002a, 2002b) findings, frontal mirror neurons function for both "understanding of goal-directed actions and for recognizing predictable visual patterns of change". They reported that serial prediction causes activation in premotor and partial cortices (see Rizzolatti, 2004). Rizzolatti refers to these authors to have explained the vital role played by the frontal mirror neuron system node regarding the representation of sequential information irrespective of whether the information is perceptual or action based. As mentioned above, communication involves both verbal and nonverbal acts, or gestures, and these data explain how participants decode the message inherent in sequential information as another nonverbal dimension.

Seemingly, it is useful in our context to refer to Minsky's explanation of sequential actions. Given the fact that he does not mention mirror neurons, Minsky (1989) provides a connectionist conception on serial actions/information processed by highly specialized agents and agencies in what he metaphorically terms "society of mind". To illustrate the concept, he enlarges upon an example of processing the act of "picking up a 
cup of tea", the endeavor for which a great number of agents (neurons like) are recruited as the same as humans are recruited in society. The example is relevant for the explanation of the sort of actions mirror neurons theorists investigate. The action presented by Minsky comprises several sub-actions:

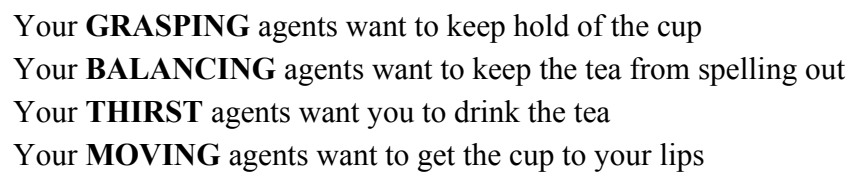

Technically, Minsky (1986) goes further with neuro-cognitive connectionist perspective introduced by Hebb (1949), revealing that neural connections and interactions constitute all mental states: thought, intelligence, memory, attention, emotion, vision, etc. Inhabiting mind-brain, specialized neuronal agents and agencies establish a vast society of neural networks. Taking this for granted, Minsky's theory (1986) also has the momentum to explain Mirror Neurons, as it were, in terms of counting them functional interconnected agents, or processors, performing specialized tasks within a mental functional unity.

Building on Minsky's seminal connectionist work and Mirror Neurons theory, neural networks reflecting the speaker's emotions, intentions and sight as well as other related mental states in an interpersonal communication, would fire (and hire) for the action of "grasping a cup of tea". Congruently, this activation applies to the receiver of the message whose mirror neurons circuits corresponding to grasping, balancing, thirst and/or moving would discharge. To make it clear, he activates the motor program used to perform the same actions. Following Keysers and Berrett (2004), activation adds information to perception in order to predict the next action. Sensory signals serve to trigger activity resonating with the sight-sound feeling attached to the action. Related to auditory and visual stimuli, the activities of the sensory set of neurons correspondingly overlap in time with the neurons of the motor that caused the action in question (see Keysers \& Berrett, 2004). Gallese and Lakoff (2005) lengthily emphasize the role of the sensory-motor system in conceptualization as well.

Drawing on Hebbian associative learning and adaptation, the neuronal circuits responding to an action, sight and sound synaptically interconnect through the spread of activation, given that they have already been potentiated through the strengthening of weights. Using Minsky's terms, the agencies mentally assigned to represent those categories are active in the interconnected communicating participant's neural networks, which, following recent studies, involve mirror neurons in premotor cortex. Accounting for the auditory and visual modalities in mirror neurons system, Rizzolatti's (2004) study shows that the audio-visual neurons code object-related actions, whereas words derive mostly from association of sound with action-neurons (i.e., echo-neurons). He relates his outcome to the "confusing organization of Broca's area in humans, where phonology, semantics, hand actions, ingestive actions and syntax are all intermixed in a rather restricted neural space", referring in his endeavor to Book Heimer's insightful findings (2002).

Another explanatory power comes from Escandell-Vidal (2009) who pinpointed that in complex activities, mirror neurons form what she terms "neural chains", which, up to a certain extent, encode particular sequences. In this connection, it seems plausible to attribute our understanding of others, when communicating, to neuronally linking our perception of their actions sequences, reflected in our mirror chains, to our memory imprints of our own past experiences; this process gives us an intuitive impression (Boumaaza, 2011) we make about them. Hence, by way of assumption, we contend that such intuition adds our idiosyncrasies alongside the 
contextual and physical elements of situations mainly studied in pragmatics (see for example, Key, 1973). Presumably, the information we add to an action relates to such intuitive mechanism in a way or another. It is our emphatic neural networks, the mechanism constituting our self-reflection, that responds to our imagination to feel what others feel, and thus we can experience emotional feedback from them (Boumaaza, 2011).

Within the framework of information addition to an action, to say, nonverbal message, and personal impression, it seems of some relevance to make reference to the sociologist and anthropologist Goffman's postulate on face security and face system's functioning in face-to-face interaction in situations where "the reciprocal influence of individuals" occurs. In his book The Presentation of Self in Everyday Life, Goffman (1959) makes explicit that when communicating, participants in an interpersonal encounter relate the received messages to their face perception as a socio-psychological construction and representation. An action might, for example, be received as a threatening act to face security. This might explain some aspects of intentional and emotional properties' reading in others and the impressions we make about them and their messages.

Using chemical language, since we are constellations of networks of chemical interactions and connections, specific neurotransmitters or chemicals are released causing mental states such as the one caused by the feeling of face threatening act. These are as follows: defensive state (norepinephrine), rewards, empowerment and self-esteem related states (Dopamine), self-belief (serotonin), affection and love (oxytocin), and so on; involved in ions flow and neurons firing, these genetic substrates explain how our neural networks relate us to others, and thus they might explain mirror neurons structural functioning.

\section{Mirror Neurons as a Common Code: Inter-subjectivity in Intra-cultural Space}

The aforementioned results drive us to think of communication as a process that comprises diverse aspects: actional, visual, auditory, emotional, kinesthetic, etc. Since face-to-face interaction constitutes one of the crucial elements of the social system that represents a significant part of individuals' socialization and life experience (Kendon, Harris, \& Key, 1975), the mentioned aspects of communication are to be taken from social neuro cognition to account for the socially formed semantic dimension.

Understanding is a central element in any communicative process. Accordingly, when we communicate, we transmit and receive meanings of the speaker's intentions and aboutness inherent in the actions or verbal acts, and we relate those to our linguistic and nonlinguistic representation. Since meanings comprise multitude dimensions: emotions, intentions and expectations, understanding such dimensions requires "shared inter-subjective space" substrated by shared neural circuits being established since our birth (Gallese, 2009) by means of associative learning postulated by Hebb (1949). Inter-subjective social space makes us plug into the whole unit (i.e., that is society) and become, up to a certain extent, symmetrical, as it were, in the socio-neuronal human network within which we identify ourselves (i.e., social identification). Neural networks integrate various neuropsychological categories (action, affect, emotions, intentions, etc.). Gallese (2009) identifies the mechanism of embodiment or embodied simulation, which functions to mediate the capacity to share meaning of those categories.

Neuro-semantic interconnectedness enables us to project our identification in interpersonal encounters on the basis of our neuronal dynamics. At the moment we communicate, by means of simulation, mirror neurons permit us to read others minds, "representing their mental specific states", including emotions, intentions, perceptions, goals, beliefs and expectations (Gallese \& Goldman, 1998); they allow us to gather information about them in order to formulate appropriate reactions. Gallese $(2007,2009)$ makes clear the mutual resonance 
of intentionally meaningful sensory "motor behavior" as the main source of knowledge we directly gather about others. He terms this mirror neurons mechanism "intentional attunement". Such mechanism is the underpinning of behaviors whose meanings are socially shared. According to Feldman Barrett's (2009) article on "connecting mind to brain", behaviors are those "actions with a meaning that is inferred by an observer". Individuals take the physical actions performed by others as meaningful behaviors referring their causes, by imputing an intention, to the actor (see also Gilbert et al., 1998; Vallacher \& Wegne, 1987) who is a social neural networking unit.

During an interpersonal exchange with an individual from another culture different from us, we act in accordance with a "set of behavioral routines and expectations". Worth noting, in this context, is that every culture sets some guiding patterns for human conduct (i.e., how one shall behave) to which linguists refer to be 'dos and don'ts'. Such patterns are internalized through the formation of mirror neurons chains (Escandell-Vidal, 2009). They compel individuals to struggle to fit in with socio-culturally set norms and get social validation for their acts and actions (Boumaaza, 2011), something which influences the shape of neural chains; thus, normative social influence equally shapes our identity and makes us socially dependent following Aronson et al (2005). It influences the formation of our semantic and semiotic dimensions of our neural circuits.

In connection to the semantic dimension, Feldman Barrett's (2009) conceptualization about what she elucidates to be ontological subjective categories might help us further understand psycho-social dimensions of intra-cultural communication and the functioning of mirror neurons. She contends that the content of a number of categories is consensually agreed upon by citizens belonging to the same country; they influence their "collective intentionality". These include what she calls nominal kind categories, that is, categories with modern psychological currency like money, marriage and nationality. She also highlights the complex psychological ones including: thought, memories, emotions and beliefs as collections of mental states produced by the brain neurons; they are constituted for phenomenological and social reasons. Worth noting in this context is that all the mentioned categories identify social and cultural groups since, following Wikan (1990), "the distinction between categories like emotion and cognition are relative". They vary from one cultural context to another (see Barrett, 2009). Seemingly, they influence our understanding of actions and behaviors within a given neuro-social space. Such ontological and psychological categories, by way of speculation and building on Hebbian "associative learning", impact the formation of mirror neurons system, which is active during interaction and communication.

\section{Communication and Mirror Neurons: Universal Dimension}

Social cognition offers scope to approach communication and interaction from a social universal perspective. It seems plausible to start discussion of this section with Feldman Barrett's (2009) conception that "some categories are cross-culturally stable since they function to serve a set of universal concerns that stem from living in large, complex groups, whereas others are culturally relative". This, together with the concept of inter-subjective space of mirror neurons circuits, helps us to briefly illuminate the universally shared neuro-social and cultural circuits.

Nowadays, we inhabit a cosmopolitan world constituting large complex groups, within which individuals lose their subjective being and their selfhood merges with humanity at large or probably with the world as whole. Tielhard Dechardin's (1955) law of complexity consciousness designates that "evolution is 
accompanied by increases in both complexity and consciousness characterized by a growing number of connections between components" (see Heylighen, 2011). The neural networks' interconnectedness, and thus mirror neurons circuits, must have been influenced, in more or less, by such complexity due to the fact that a world of divers "economic and psychic affiliations is being woven", a fact which no one can ignore; such affiliations constantly and deeply envelop and penetrate every single individual. Building on Theilhard (1969), Heylighen notes that no one can "act or think otherwise than collectively". Such collectivity is the result of a whole complex adaptive dynamic system that is human civilization culminating in a complex functional unity of neural networks.

Struzik (2015) relates the functional unity of neural networks, comprising mirror neurons, to "the tools we create, including the 'instruments' of our societal, religious and cultural systems". Commensurably, Heyes (2005) argues that mirror neurons system is a product (as well as a process) of social interaction. The associative account implies that mirror neurons come from sensorimotor experience, and that much of this experience is obtained through interaction with others. Accordingly, universal culture is propagated by the mainstream media' circuits and educational curricula, fashion, food, and so on, while the decrease of emotional differences entropy; this promotes a single way of thinking using roughly the same semiotic code to which Heylighen refers as "super-consciousness" that envelops the globe.

In the same vein, Aldous Huxley's artistic work prophesized a long time ago a world in which psycho-neurological differences of the population are ironed out and standardized in the age of post-genomics and prevalence of hedonism as a predominant element of cultural catalogue; this is through regulating proteins underpinning our behaviors including the rewarding protein "dopamine", etc. Thus, he inadvertently indicates to mirror neurons synchronization in his envisioned "Brave New World". In such world humans act, visualize and understand actions and intentions in roughly the same way within the whole social network, whose instruments activate selected behaviors, actions and emotions referring to one all-embracing cultural catalogue. A Hollywood movie would find its way in the socio-psychological matrix to influence people all over the world; its communicated actional and behavioral messages can activate mirror neurons of people connected to the emotions of the movie's characters; as a result, viewers sympathize with them because they have established for such purpose mirror neurons chains throughout their life experience. Taking into consideration that through the formation of millions of stable chains of mirror neurons, consumerist culture has long aimed at changing self-image, which is a hallmark characteristic of our age.

\section{Conclusion}

Grounding mainly on the framework of mirror neurons and relatively that of neural networks, we highlighted the neural underpinnings of nonverbal communication. In the first section, we illuminated numerous aspects of communicational behavior in its interpersonal dimension: actional, auditory, visual, emotional, and intentional patterns and how these are linked to mirror neurons active in interpersonal interaction. We made reference to Hebbian rule and minsky's connectionist conception to further illustrate our lines of argumentation. The second section lent heavy importance to the neurocognitive underpinnings of communication in its socio-cultural dimension, focusing mainly on the semantic element; we pinpointed the extent to which such dimension reflects the shared neural circuits that enable individuals in a given cultural space to connect to each other within their collectively established neuro-social space. The third section placed particular importance on the universal dimension of communication arguing that mirror neurons and neural 
networks have been synchronized, and, thus, understanding of actions and behaviors have been standardized.

\section{References}

Anthony, H. N. (2016). Handbook of research on effective communication, leadership, and conflict resolution. USA: California State University, Dominguez Hills.

Aronson, E., Wilson, T. D., \& Akert, A. M. (2005). Social psychology (5th ed.). Upper Saddle River, NJ: Prentice Hall.

Barrett, L. F. (2009). The future of psychology: Connecting mind to brain. Perspectives in Psychological Science, 4, 326-339.

Boumaaza, B. (2011). Athene's theory of everything. Reese Leysen et al. (Eds.).

Brown, R. E., \& Milner, P. M. (2003). The legacy of Donald O. Hebb. More than the Hebb synapse. Nat Rev Neurosci, 4 , 1013-1019.

Doran, D. M., \& McNeilage, A. (1998). Gorilla ecology and behaviour. Evolutionary Anthropology, 6, 120-131.

Escandell-Vidal, V (2009). Social cognition and intercultural communication. In V. Guillén-Nieto, C. Marimón-Llorca, and C. Vargas-Sierra (Eds.), Intercultural business communication and simulation and gaming methodology (pp. 65-96). Bern: Peter Lang.

Gallese, V., \& Lakoff, G. (2005). The brain's concepts: The role of the sensory-motor system in conceptual knowledge. Cognitive Neuropsychology, 22, 455-479.

Gallese, V. (2006). Intentional attunement: A neurophysiological perspective on social cognition and its disruption in autism. Brain Research, 1079, 15-24.

Gallese, V. (2009). Mirror neurons, embodied simulation, and the neural basis of social identification. Psychoanalytic Dialogues, 19, 519-536.

Gallese, V., \& Goldman, A. (1998). Mirror neurons and the simulation theory of mind-reading. Trends in Cognitive Sciences, 3 , 493-501.

Gallese, V., Fadiga, L., Fogassi, L., \& Rizzolatti, G. (1996). Action recognition in the premotor cortex. Brain, 119, 593-609. Oxford: Oxford University Press.

Gilbert, T., Pinel, C., Wilson, D., Blumberg, J., \& Wheatley, P. (1998). Immune neglect: A source of durability bias in affective forecasting. Journal of Personality and Social Psychology, 75(3), 617-638.

Hebb, D. (1949). The organization of behavior. New York: Wiley \& Sons.

Heyes, C. M. (2005). Imitation by association. In S. Hurley and N. Chater (Eds.), Perspectives on imitation: From mirror neurons to memes. Cambridge: MIT Press.

Heylighen, F. (2011). Conceptions of a global brain: An historical review. Evolution: Cosmic, biological, and social. Volgograd, Russia: Uchitel Publishing.

Huxley, A. (1946). Brave new world. New York: Harper \& Row.

Kendon, A., Harris, R. M., \& Key, M. R. (1975). Organization of behavior in face-to-face interaction. Mouton Publisher, Chicago: Walter de Gruyter.

Key, M. R. (1973). Nonverbal communication in speech acts. Paper presented at Conference on Sociology of Language and Theory of Speech Acts, University of California, Irvine.

Keysers, C. (2011). The empathic brain. Groningen: Social Brain Press.

Keysers, C., \& Perrett, D. I. (2004). Demystifying social cognition: A Hebbian perspective. Trends in Cognitive Sciences, 8(11), 501-550.

Keyton, J. (2011). Communication and organizational culture: A key to understanding work experience. Thousand Oaks, CA: Sage.

Lévy, P. (1997). Collective intelligence: Mankind's emerging world in cyberspace. New York: Plenum.

Löwel, S., \& Singer, W. (1992). Selection of intrinsic horizontal connections in the visual cortex by correlated neuronal activity (pp. 209-212). United States: American Association for the Advancement of Science.

Meister, I. G., Boroojerdi, B., Foltys, H., Sparing, R., Huber, W., \& Topper, R. (2003). Motor cortex hand area and speech: Implications for the development of language. Neuropsychologia, 41, 401-406.

Mesquita, B., Barrett, L. F., \& Smith, E. (Eds.). (2010). The mind in context. New York: Guilford.

Minsky, M. (1986). The society of mind. New York: Simon and Schuster.

Molnar-Szakacs, I., \& Overy, K. (2006). Music and mirror neurons: From motion to "e"motion. Social Cognitive and Affective Neuroscience, 1(3), 235-241.

Richard, E. B., \& Peter, M. M. (2003). Timeline: The legacy of Donald O. Hebb: More than the Hebb synapse. Nature Reviews 
Neuroscience, 4, 1013-1019.

Rizzolatti, G., \& Craighero, L. (2004). The mirror-neuron system. Annual Review of Neuroscience, 27(1), 169-192.

Rizzolatti, G., Fogassi, L., \& Gallese, V. (2001). Neurophysiological mechanisms underlying the understanding and imitation of action. Nat Rev Neurosci, 2, 661-670.

Schiaratura, L. T., Pastena, A. D., Askevis-Leherpeux, F., \& Clément, S. (2015). Verbal and gestural communication in interpersonal interaction with Alzheimer's disease patients. Geriatr Psychol Neuropsychiatr Vieil, 13(1), 97-105.

Schubotz, R. I., \& Von Cramon, D. Y. (2001). Interval and ordinal properties of sequences are associated with distinct premotor areas. Cerebral Cortex, 11, 210-222.

Schubotz, R. I., \& Von Cramon, D. Y. (2002a). Predicting perceptual events activates corresponding motor schemes in lateral premotor cortex: An fMRI study. Neuro Image, 15, 787-796.

Schubotz, R. I., \& Von Cramon, D. Y. (2002b). A blueprint for target motion: fMRI reveals perceptual complexity to modulate a premotor-parietal network. Neuro Image, 16, 920-935.

Vallacher, R. R., \& Wegner, D. M. (1987). What do people think they're doing? Action identification and human behavior. Psychological Review, 94, 3-15. 